\section{¿EXISTE LA EQUIDAD INJUSTA? POR LA ELIMINACIÓN DEL REQUISITO OBLIGATORIO DEL SERUMS}

\author{
IS THERE UNJUST EQUITY? FOR THE \\ ELIMINATION OF THE MANDATORY SERUMS \\ PROGRAM (RURAL AND URBAN MARGIN \\ HEALTH CARE SERVICE)
}

\begin{abstract}
Percy Mayta-Tristán ${ }^{1, a}$, Julio A. Poterico ${ }^{2, b}$, Edén Galán-Rodas ${ }^{3, a}$, Daniel Raa-Ortiz ${ }^{4,5, c}$
\end{abstract}

Sr. Editor. Hemos leído la respuesta de Mujica et al. a nuestro manuscrito sobre el requisito obligatorio del servicio social en salud (SERUMS) ${ }^{(1)}$. Ellos basan su comentario sobre uno de los aspectos introductorios del artículo (la búsqueda de evidencias sobre el efecto de la intervención) y no sobre el fondo de nuestra propuesta, ya que mencionan que la "inefectividad de un programa social no lo hace necesariamente inconstitucional (i.e., falacia non sequitur)". Este comentario nos llama la atención porque en ninguna parte del manuscrito hemos afirmado que la inconstitucionalidad se deba a la falta de evidencias de efectividad del programa, sino al requisito obligatorio del SERUMS para que los recursos humanos en salud (RHUS) puedan ingresar a programas de segunda especialización, ocupar cargos en entidades púbicas, recibir becas u otra ayuda equivalente para estudios o perfeccionamiento ${ }^{(2)}$. Nosotros basamos nuestro análisis de inconstitucionalidad en que este requisito obligatorio afecta tanto el derecho al trabajo y el derecho a la educación, y que por ser aplicado solo para los RHUS, es discriminatorio.

Sobre el análisis ecológico presentado por los autores, un reciente artículo publicado por la Dirección General de Recursos Humanos del Ministerio de Salud (MINSA) muestra lo mismo, pero con datos recogidos en forma primaria. Encontraron que si bien aún no se llega al número mínimo recomendado de RHUS en las regiones, se tiene una distribución más equitativa de

\footnotetext{
1 Escuela de Medicina, Universidad Peruana de Ciencias Aplicadas. Lima, Perú.

2 Puesto de Salud Santa Cruz de Ratacocha, Ministerio de Salud. Huánuco, Perú.

3 Escuela de Medicina y Cirugía, Universidad Hispanoamericana. San José, Costa Rica.

4 Estudio Trujillo Amoros. Lima, Perú.

Facultad de Derecho, Universidad del Rosario. Rosario, Argentina.

Medico salubrista; ${ }^{\mathrm{b}}$ médico serumista; ${ }^{\mathrm{c}}$ abogado constitucionalista Recibido: 02-03-15 Aprobado: 04-03-15
}

Citar como: Mayta-Tristán P, Poterico JA, Galán-Rodas E, Raa-Ortiz D ¿Existe la equidad injusta? por la eliminación del requisito obligatorio de SERUMS [carta]. Rev Peru Med Exp Salud Publica. 2015;32(1):195-6. los mismos ${ }^{(3)}$, y mencionan que una de las posibles razones son las políticas de expansión del SERUMS. Como hemos discutido previamente en nuestro artículo, el SERUMS permite distribuir RHUS a zonas vulnerables, pero falla en la retención de estos. Por ello, expusimos como ejemplo el modelo chileno que busca no solo distribución, sino también retención de RHUS (4).

Finalmente, los datos presentados por Mujica et al. y por el MINSA (3), son parte de las escasas evidencias recientemente disponibles sobre el SERUMS, y abogan por la contribución de este programa a una distribución menos inequitativa del personal de salud. La inequidad por definición es una desigualdad injusta y evitable, es decir, que se pueden conocer las causas de la desigualdad y fundamentar un juicio sobre la injusticia de dichas causas ${ }^{(5)}$. Ante esto nos preguntamos ¿existe una equidad injusta?, el SERUMS promueve la equidad de la distribución de los RHUS a costa de una obligatoriedad que atenta contra los derechos al trabajo y a la educación. Esta medida es injusta porque es discriminatoria (solo se aplica a RHUS) y porque existen evidencias de otras estrategias para lograr distribuir y retener RHUS en zonas vulnerables que no son basadas en la obligatoriedad ${ }^{(4)}$.

Por ello, nos reafirmamos en la necesidad de eliminar el requisito obligatorio del SERUMS para acceder a cargos públicos o programas de segunda especialización, u alguna medida similar, y que el MINSA formule estrategias que hagan atractivo el trabajo en zonas vulnerables para los RHUS. Se debe buscar situaciones de equidad en todos los derechos constitucionales, tanto para la población en el acceso a salud, como para los profesionales de la salud en el acceso al trabajo y a la educación.

Conflictos de interés: PMT, EGR han realizado el SERUMS, JAP está realizando el SERUMS y DRO no realizó servicio social porque para los abogados no es obligatorio.

\section{REFERENCIAS BIBLIOGRÁFICAS}

1. Mayta-Tristán P, Poterico JA, Galán-Rodas E, Raa-Ortiz D. El requisito obligatorio del servicio social en salud del Perú: discriminatorio e inconstitucional. Rev Peru Med Exp Salud Publica. 2014;31(4):781-7.

2. Perú, Congreso de la República. Decreto Ley 23330 Ley del Servicio Rural y Urbano Marginal de Salud - SERUMS. Lima: Congreso de la República; 1981.

3. Moscoso Rojas B, Huaman Angulo L, Nuñez Vergara M, Llamosas Felix E, Perez W. Inequidad en la distribución de recursos humanos en los establecimientos del Ministerio de Salud de cuatro regiones del Perú. An Fac Med (Lima). 2015;76(1):35-40.

4. Peña S, Ramirez J, Becerra C, Carabantes J, Arteaga O. The Chilean Rural Practitioner Programme: a multidimensional 
strategy to attract and retain doctors in rural areas. Bull World Health Organ. 2010;88(5):371-8.

5. Schneider MC, Castillo-Salgado C, Bacallao J, Loyola E, Mujica OJ, Vidaurre M, et al. Métodos de medición de las desigualdades de salud. Rev Panam Salud Publica. 2002;12(6):398-415.

Correspondencia: Percy Mayta-Tristán

Dirección: Av. Brasil 2169, Dpto 802, Jesús María, Lima, Perú.

Teléfono: (51) 987532133

Correoelectrónico:percy.mayta@upc.edu.pe

\section{MEDICIÓN Y MAGNITUD DEL BULLYING EN PERÚ}

\section{MEASUREMENT AND MAGNITUDE OF BULLYING IN PERU}

\section{Carla Cobián-Lezama1,a, Ayar Nizama-Vía ${ }^{1,2, a}$, David Ramos-Aliaga ${ }^{1, a}$, Percy Mayta-Tristán ${ }^{1, b}$}

Sr. Editor. El bullying es un problema de salud pública que incluye a tres actores: la víctima, el victimario/ agresor y el observador, y es un hecho que está cada vez más presente en nuestra sociedad, pues se discute sus implicancias en escenarios de salud, educación y hasta en la prensa. Sin embargo, la información científica disponible sobre la magnitud del bullying en el Perú (Tabla 1) muestra prevalencias bastante elevadas y hasta alarmantes, por lo que es válido preguntarse si es que el bullying en el Perú se está midiendo de manera correcta.

La definición del bullying victimización incluye tres características que deben cumplirse siempre: debe haber un desbalance de poder en favor del agresor, debe existir una intención de hacer daño y, finalmente, debe ser sistemático; es decir, repetido en el tiempo (1). Por ello, las escalas que se usan para medirlo deben tener en cuenta estos aspectos, de lo contrario se podría

\footnotetext{
1 Escuela de Medicina, Universidad Peruana de Ciencias Aplicadas. Lima, Perú

2 Sociedad Científica de Estudiantes de Medicina, Universidad Peruana de Ciencias Aplicadas. Lima, Perú

a Estudiante de medicina; ${ }^{\text {b }}$ Médico salubrista

Recibido: 09-10-14 Aprobado: 15-10-14
}

Citar como: Cobián-Lezama C, Nizama-Vía A, Ramos-Aliaga D, MaytaTristán P. Medición y magnitud del bullying en Perú [carta]. Rev Peru Med Exp Salud Publica. 2015;32(1):196-7. cometer un error al medir agresiones esporádicas y catalogarlas como bullying.

Al revisar la bibliografía peruana sobre estudios de bullying se ha evidenciado que los diferentes autores utilizan básicamente dos escalas para evaluar este fenómeno, estas son la escala de DEVIDA (2) y la de Oliveros ${ }^{(3)}$. La escala de DEVIDA en su versión 2012 incluye 14 situaciones de violencia durante el último año y considera que cuando presenta tres o más características hay bullying moderado. Oliveros generó una escala en el 2007 que evalúa 9 situaciones de violencia durante el último año y considera bullying cuando presenta dos o más características. Ambas escalas no consideran el elemento sistemático como parte de la definición de bullying, además, ninguna cuenta con un estudio formal de validación, por lo tanto, no existe garantía de que midan realmente bullying.

Incluso, en algunos casos a pesar de usar la misma escala, algunos autores han creado definiciones operativas distintas, por lo que los valores de prevalencia reportados no miden lo mismo (Tabla 1), pues usan diferente número de situaciones de violencia, y las temporalidades (último año, durante la secundaria, sin mención de la temporalidad) son diferentes, por lo que la magnitud del fenómeno bullying en los estudios peruanos no es clara. Por ello, existe una diferencia grande entre los reportes de países europeos donde la prevalencia de bullying varía entre 10 a $20 \%{ }^{(4)}$ y los estudios peruanos entre 35 a $60 \%$.

Esta situación impide la comparación entre estudios, y sus prevalencias, lo que dificulta un diagnóstico global y limita la posibilidad de desarrollar estrategias o asignar recursos para intervenciones futuras en las poblaciones más afectadas. Así mismo, el contar con una medición estándar permitiría evaluar en el tiempo el impacto de dichas intervenciones y elegir las más efectivas.

La comunidad científica que estudia el bullying en el Perú, debe generar escalas que incluyan el comportamiento sistemático y una temporalidad clara, o traducir y adaptar culturalmente escalas generadas en inglés que además permitirían compararnos con otras realidades ${ }^{(5)}$. De esta manera se podrá tener una prevalencia más certera que refleje la realidad en nuestro país.

Conflictos de interés: no existen conflictos de interés en la publicación de este artículo 